\title{
Identification of the Clinical Predictors of Mortality in Blunt Polytrauma Patients Attending the Emergency Department in Suez Canal University Hospital
}

\author{
Zeinab M. Abd EL-Atiff', Adel H. El-Baih ${ }^{1^{*}}$, Ahmed E. Abou Zeid', Goda M. \\ Ellabban $^{2}$
}

Departments of ${ }^{1}$ Emergency Medicine, ${ }^{2}$ General Surgery, Faculty of Medicine, Suez Canal University, Egypt

\begin{abstract}
Background: Trauma is a worldwide problem. It is the disease of young and the leading cause of death in the first four decades of life. Several trauma scores have been devised to predict injury severity and risk of mortality. Trauma score systems try to translate the severity of injury into a number. Regardless their accuracy, they are complex to calculate and it is clear that their use in clinical decision making is limited. Aim: The aim of this study was to identify the clinical predicting factors of mortality in blunt poly-trauma patients. Patients and methods: This was a descriptive study conducted on 106 adult blunt poly-traumatized patients from July 2013 to December 2013 attending the Emergency Department at Suez Canal University Hospital. Results: In this study, $78.3 \%$ of 106 patients were males. Mortality rate was $18.9 \%$, motor car accidents were responsible for $87.7 \%$ of the total cases. The most common sites to be affected were the extremities and pelvis but the head was the only injury related to mortality shock index (SI) was the most sensitive predictor $100 \%$ and the most specific one was ISS $100 \%$. Heart rate (HR), Glasgow Coma Score (GCS), and systolic blood pressure (SBP) were good predictors to mortality. Conclusion: We conclude that SI, modified shock index (MSI), injury severity score (ISS), revised trauma score (RTS), and vital signs with presence of head trauma and the need for intubation or ICU admission are predictors of mortality in poly-traumatized patients.
\end{abstract}

Keywords: multiple-trauma, clinical stratification, ICU

\section{Introduction}

Trauma is an injury to the body that occurs when an uncontrolled force or acute source of energy makes contact with the body and the body cannot tolerate $\mathrm{it}^{(1)}$. Blunt injuries are a type of mechanical trauma caused by impact or other force applied from or with a blunt object ${ }^{(2)}$. Polytrauma is defined as two or more injuries that occur simultaneously and affect differ- ent organs and body systems occurring. One of which may be life-threatening, resulting in physical, cognitive, psychological, or psychosocial impairments and disability $^{(3,4)}$. Trauma is a worldwide problem, with severe and wide-ranging consequences for individuals and society as a whole and accounts for a significant proportion of annual deaths worldwide. Trauma is the sixth leading cause of death worldwide, accounting for $10 \%$ of all mortalities and is 
still the most frequent cause of death in the first four decades of life ${ }^{(5,6)}$. The care of acutely injured people is a public health system concern, which involves health care professionals and health care systems. It encompasses pre-hospital assessment and care by emergency medical services personnel, emergency department assessment, treatment, stabilization and inhospital care among all age groups ${ }^{(7,8)}$. In order to minimize the mortality rate associated with trauma, the factors affecting mortality should be evidenced to take care of patients. In the late years, several statistical models such as Trauma and Injury Severity Score [TRISS] and New Injury Severity Score [NISS] have been developed in an attempt to accurately predict outcomes in trauma patients ${ }^{(9,10)}$. The anatomic portion of these models makes them difficult to use when performing a rapid initial trauma assessment that helps us to predict the outcome and mortality of the patients ${ }^{(11)}$. So the purpose of this study is to find out the clinical risk factors that can help to predict mortality and improve the outcome of the patients including age, gender of the patients, mechanism of injury, vital signs, shock index, modified shock index, injury severity score and revised trauma score.

\section{Patients and Methods}

This is a cross sectional descriptive study that had been carried on all the blunt polytrauma patients attended to the Emergency Department at Suez Canal University Hospital from July 2013 to December 2013. The inclusion criteria were: 1) Adult patients (18 years old and more), 2) Both sexes, 3) Patients with injury to several physical regions or organ systems, where at least one injury or the combination of several injuries are life threatening. Patients transferred from other hospitals after any medical or surgical procedures, having burn, associated blunt and penetrating trauma or patients who had systemic diseases were excluded from this study. Data was collected in a pre-organized data sheet for each patient including demography, clinical assessment according to the ABCDE approach, cause of injury, duration prior to admission, vital signs at admission, Glasgow coma scale at admission, shock index, modified shock index, revised trauma score, injury severity score, associated injuries, type of management instituted, $H G$, HTC, blood transfusion if needed and the final outcome of the patients as: Died, Admitted to ICU, Operative intervention \& result of this intervention or admitted to inpatient department.

\section{Results}

This study was conducted on 106 patients to assess the clinical predictors of mortality in blunt poly-trauma patients attending the Emergency Department in Suez Canal university hospital. Out of 106 patients, 86 (81.1\%) patients survived. Age distribution ranged from 19 - 59 years old.There were 83 males and a male to female ratio of 3.6: 1 (Table 1).

Table 1: Age and gender distribution of the studied patients $(n=106)$

\begin{tabular}{|c|c|c|c|}
\hline \multicolumn{2}{|c|}{ Characteristic } & Number & Percent \\
\hline \multirow{3}{*}{ Age@ } & $<20 \mathrm{y}$ & 8 & $7.6 \%$ \\
(Yrs) & $20-29 \mathrm{y}$ & 39 & $36.8 \%$ \\
& $30-39 \mathrm{y}$ & 19 & $17.9 \%$ \\
& $40-49 \mathrm{y}$ & 24 & $22.6 \%$ \\
& $50-59 \mathrm{y}$ & 16 & $15.1 \%$ \\
\hline \multirow{2}{*}{ Gender } & Male & 83 & $78.3 \%$ \\
& Female & 23 & $21.7 \%$ \\
\hline
\end{tabular}

@ Mean \pm SD $=33.7 \pm 11.9$ (range, $19-59)$

Mortality was maximum in patients $>49$ years old (43.8\%) with statistically significant relation between increasing age and mortality. The study showed that most of 
the patients arrived to the hospital between 30- 60 minutes (70.7\%) and that delay in arrival to hospital had significant association with mortality. The study revealed that motorcar accidents were responsible for $87.7 \%$ of the total cases; followed by $8.5 \%$ cases due to falling from height with only $3.8 \%$ due to violence. There was no statistically significant relation between mechanism of injury and mortality. The study showed that the extremities and pelvis injuries were the most common sites to be affected by blunt trauma (72\%). The head is the only injury showing significant association with mortality $(p<0.05)$ in contrast to other injuries $(p>0.05)$. The study revealed that heart rate, systolic blood pressure and Glasgow coma scale were good predictors of mortality: the most sensitive one was the heart rate $75 \%$ and the most specific one was systolic blood pressure $74.4 \%$ as shown in table 2 . The study revealed that shock index; modified shock index, injury severity score and revised trauma score showed a statistically significant difference between sur- vived and deceased patients. The most sensitive predictor was shock index 100\% while the most specific predictor was injury severity score $100 \%$ as shown in table 3 . The study showed that $\mathrm{HB}, \mathrm{HCT}$, and number of units needed for blood transfusion are good predictors of mortality with statistically significant difference between deceased and survived patients. Also it showed that the majority of patients (72.6\%) had been subjected to conservative treatment with statistically significant difference between deceased and survived patients. The study revealed that $48.1 \%$ of the patients needed ICU admission, 30.2\% needed endotracheal intubation, and both of them had significant association for mortality. The study showed that most of the studied patients have been admitted to inpatient ward under observation (39.6\%) or after surgical intervention (10.4\%). While (31.1\%) of the patients have been admitted to ICU either directly after trauma (24.5\%) or may be after surgery (6.6\%), while mortality rate was $18.9 \%$ as shown in figure 1.

Table 2: Comparison between heart rate, systolic blood pressure, respiratory rate and Glasgow coma scale regarding specificity and sensitivity

\begin{tabular}{|l|ccccc|}
\hline Vital Signs & AUC & p-value & Cutoff value & Sensitivity & Specificity \\
\hline Heart Rate & 0.870 & $0.000^{*}$ & 127 & $75 \%$ & $12.8 \%$ \\
Systolic Blood Pressure & 0.626 & $0.01^{*}$ & 89 & $45 \%$ & $74.4 \%$ \\
Respiratory Rate & 0.303 & 0.3 & 22 & $60 \%$ & $37.2 \%$ \\
Glasgow Coma Scale & 0.734 & $0.005^{*}$ & 9 & $40 \%$ & $72.1 \%$ \\
\hline
\end{tabular}

* Statistically significant $(p$-value $<0.05)$

Table 3: Comparison between SI, MSI, IS and RTS regarding specificity and sensitivity

\begin{tabular}{|l|ccccc|}
\hline \multicolumn{1}{|c|}{ Clinical Predictors } & AUC & $p$-value & Cutoff value & Sensitivity & Specificity \\
\hline Shock index & 0.999 & $<0.001^{*}$ & 1.55 & $100 \%$ & $98 \%$ \\
Modified shock index & 0.987 & $<0.001^{*}$ & 1.55 & $95 \%$ & $89 \%$ \\
ISS & 0.930 & $<0.01^{*}$ & 39 & $85 \%$ & $100 \%$ \\
RTS & 0.860 & $0.03^{*}$ & $\geq 6$ & $75 \%$ & $71 \%$ \\
\hline
\end{tabular}

* Statistically significant ( $p$-value $<0.05$ ) 


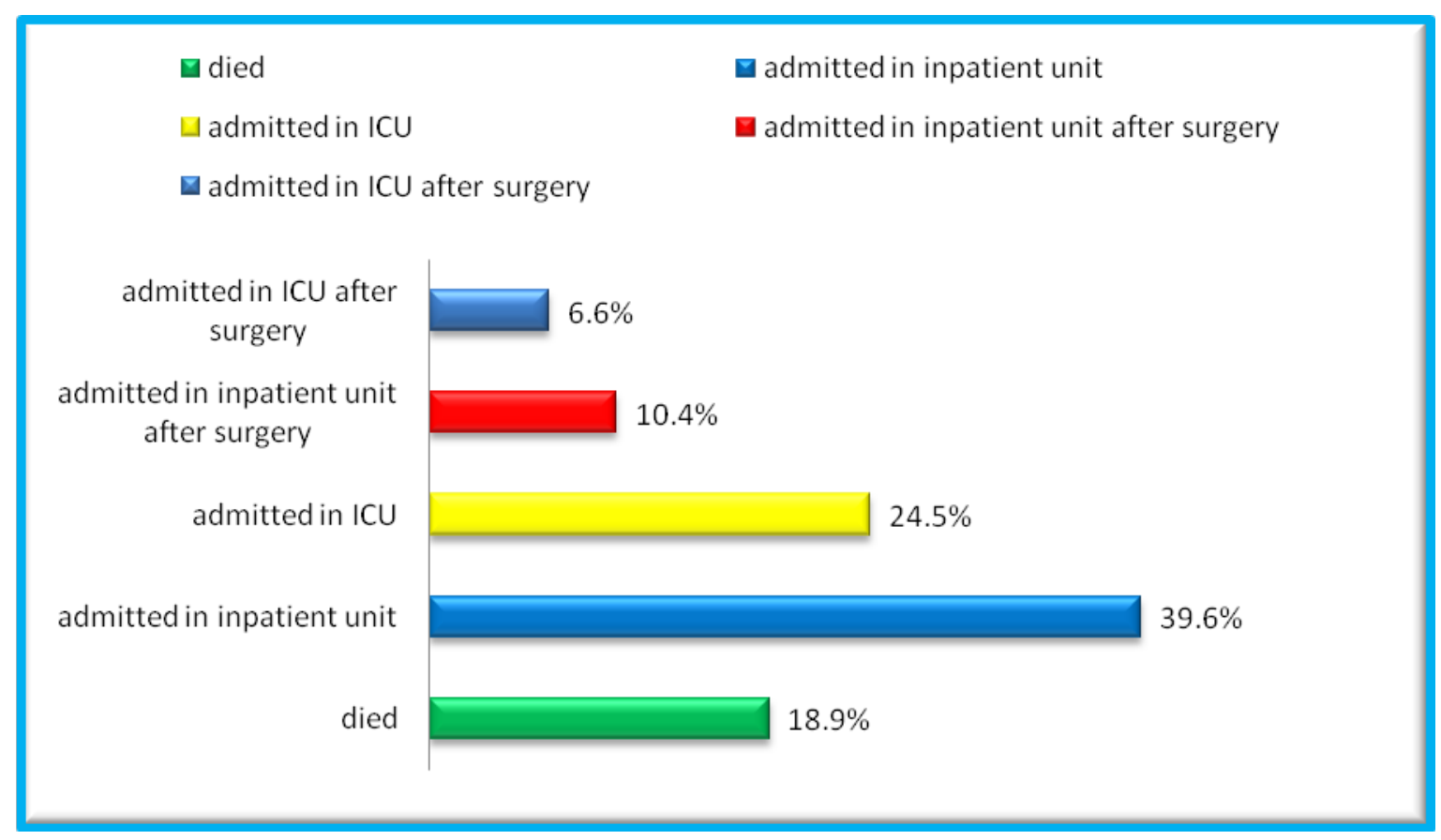

Figure 1: Distribution of the studied patients is according to their outcome $(n=106)$

\section{Discussion}

It is a well-known fact that trauma mostly affects the youth, particularly the third and fourth decades ${ }^{(12,13)}$. In our study, the mean age was $33.7 \pm 11.9$ year, $55 \%$ were in age group between $20-40$ years and males comprised (78\%.3) of the patients. Our results are comparable with a study done in India by Murlidhar $\mathrm{V}$ et al, in which most of the patients were men (84\%) and the average age was 31 years $^{(8)}$. This agrees with a study by Totterman A et al, where $76 \%$ of the victims were males with a mean age of 36.6 years $^{(9)}$. In our study, out of 106 patients 20 patients died (18.9\%). Our result was very far from mortality rates mentioned in international studies $(4-6 \%)^{(14,16)}$. This is due to the difference in the trauma management strategies starting from first aid to definitive management and lack of understanding and application of the trauma protocols in Egypt. This also may be due to our selection to severe poly-traumatized patients, delay in transfer and lack of ICU beds. Our results agree with Curtis K et al study in which 507 severely injured patients out of them 100 patients (19.7\%) died ${ }^{(11)}$. Also mortality rate was $11 \%$ in study conducted by Copes et al which is close to our results $^{(12)}$. Sogut $O$ et al, had studied the Injury trends and mortality in adult patients with major trauma in New South Wales. They found that the percentage of in hospital deaths were $13.6 \%$ when severe polytraumatized patients were selected ${ }^{(13)}$. Regarding the effect of age on mortality, in our study the mortality rate increases with age as $43 \%$ of death occurred in patients in age group (50-60) with statistically significant relation $(\mathrm{P}=0.001)$. Copes $\mathrm{W}$ et al, in a large study showed that mortality becomes nearly the double for patients over 50 years of age with the same degree of injury severity as compared to patients below 50 years of age ${ }^{(12)}$. Our results agree with Hsiao $\mathrm{K}$ et al in which the mortality rate increased with age and was $44.8 \%$ for those $45-59$ years ${ }^{(15)}$. Similar results were found in another study in India where mortality was maximum in patients of age group $>50$ years $^{(9)}$. Regarding the time interval be- 
tween trauma and hospital arrival, we found that $70.8 \%$ of the patients arrived to the hospital 30-60 minutes after the trauma event. This agrees with Hsiao $\mathrm{K}$ et al in which the median time to hospital arrival was 45 minutes $^{(15)}$. Singh $\mathrm{J}$ et al estimated that time delay between the injury and the start of definitive treatment is vital to the outcome. They found that there was a proportionate increase in mortality with increase in delay in arrival ${ }^{(14)}$. In agreement with our results which found that delay in arrival is strongly related with percent of mortality. In our study motor car accidents were responsible for $87.7 \%$ of the total cases followed by falls $8.5 \%$. This agrees with several studies conducted on trauma epidemiology, where the majority of cases were due to road side collisions ${ }^{(6,9)}$. Singh J et al had found that Road traffic collisions were the most common cause of trauma $(72 \%)^{(14)}$. Another study, conducted by Nastaran HK et al in which the cause of injuries was road traffic accidents (74.2\%), followed by falls $(12.1 \%)^{(17)}$.

In our study extremities and pelvis injuries were the most common sites to be affected by blunt trauma (72\%) but the head was the only injury showing significant association with mortality $(p<0.05)$ in contrast to other injuries $(p>0.05)$ as head trauma presented in $75 \%$ of died patients. Our results agree with Ustundag et al. who estimated that serious head injury was present in $8.9 \%$ and $70.3 \%$ of the patients who survived and died respectively. The presence of serious head injury was found to be statistically significant with respect to mortality, which was consistent with other studies in the literature ${ }^{(18)}$. Head trauma, leading to mortality and morbidity, has become a serious health problem in industrialized countries, the cause of which was commonly the result of traffic accidents ${ }^{(19)}$. Our results agree also with Tinkoff $G$ et al study in which head trauma present in $84 \%$ of died patients ${ }^{(20)}$. Also Rabbani $A$ et al who estimated that mortality was strongly related to severe head injury in traumatic ICU patients $^{(21)}$. We found a statistically significant difference in HR, SBP and GCS between the deceased and survived patients $(p<0.05)$. This is in agreement with the results of a study conducted by Hannan al, in which $\mathrm{HR}>120$ beats/min, $\mathrm{SBP}<90 \mathrm{~mm} \mathrm{Hg}$ and $\mathrm{DBP}<60 \mathrm{mmHg}$ correlated with the mortality rate ${ }^{(22)}$. Similarly Guyette $\mathrm{F}$ et al, found that abnormal GCS was found to be a strong predictor of death ${ }^{(18)}$. In another prospective cohort study in adults ( $\geq 16$ years) conducted by Hasler RM et al in which SBP below $110 \mathrm{mmHg}$ was found to be associated with increased mortality in major trauma patients, mortality was doubled at SBP 90-109mmHg, four-folds higher at $70-89 \mathrm{mmHg}$ and 10 -fold higher at $<70 \mathrm{mmHg}$. SBP $\geq 150 \mathrm{mmHg}$ were associated with decreased mortality ${ }^{(19)}$. A study done by Sogut $O$ et al demonstrated that a significantly higher mortality rate was associated with $\mathrm{SBP}<90 \mathrm{mmHg}$ and GCS score $<8^{(13)}$. Bilgin NG et al, found that when cut off value was taken 6.60 , the success of RTS in distinguishing traumatized patients in a life threatening condition (specificity) was found $74.3 \%$, and its success in distinguishing traumatized patients in a non-life threatening condition (sensitivity) was found $54.8 \%$. As RTS was found to have fair sensitivity but good specificity, it would be more appropriate to use RTS for distinguishing life threatening injuries ${ }^{(23)}$. It is similar to our study where it was found that when the cut off value was taken 6 , specificity was $71 \%$ while sensitivity was $74 \%$. In contrast to our study results, Liu $\mathrm{Y}$ et al revealed that RTS had sensitivity $88 \%$ and specificity $90 \%$ and according to these results, it can be stated that the revised trauma score is helpful in classification of traumatic patients and prediction of their mortality ${ }^{(17)}$. King PM et al reported various 
limitations of injury severity score (ISS). First, the ISS often leaves some injuries out of the scoring process altogether, such as when a patient sustains multiple injuries to a single body region, as it considers at most one injury per body region, Second, it takes no account of physiological variables, such as alcohol and drug consumption, pregnancy, and systemic diseases ${ }^{(24)}$. Bilgin et al, reported an ISS sensitivity of $85.7 \%$ and specificity of $99.2 \%$ at a cut off value of $19^{(23)}$. It is similar to our study results in which specificity of ISS was $100 \%$, sensitivity was $85 \%$ when cut off point of ISS accepted as 39. As regard to shock index (SI) Guyette $\mathrm{F}$ et al, showed that SI had a sensitivity of $43 \%$ and a specificity of $89 \%$ when a cut-off point of 0.88 is used ${ }^{(18)}$. In contrast to our results SI had a specificity of $98 \%$ and a sensitivity of $100 \%$ when the cut-off value is 1.5 . Regarding the modified shock index (MSI); our study results revealed that it had a specificity of $89 \%$ and a sensitivity of $95 \%$ but no previous data exists. Concerning the comparison of the four predictors in our study (RTS, ISS, SI and MSI) SI had a good combination of high specificity and sensitivity. Comparable performances of the RTS, ISS, SI and MSI showed that the RTS as the poorest. In our study, the mean hemoglobin concentration for the dead patients was $9.27 \pm 1.2 \mathrm{~g} / \mathrm{dl}$ where the mean hemoglobin concentration for the survived was $10.7 \pm 1.7 \mathrm{~g} / \mathrm{dl}$ and there was statistically significant difference between survived and dead patients regarding hemoglobin $(p=0.01)$. This met the results of a Canadian study that revealed that a mean hemoglobin concentration $<9 \mathrm{~g} / \mathrm{dl}$ was independently associated with an increased risk of hospital mortality ${ }^{(24)}$. Regarding the intubation and need for ICU, there were significant relation to mortality in our study as $60 \%$ of deceased patients were intubated and $90 \%$ of died patients need ICU. Our result agree with the study of Hasler R, which revealed that intubation or cardiopulmonary resuscitation was initiated in $88 \%$ of those who died and $43.5 \%$ of those who survived $(p<0.05)^{(19)}$. Hannan $L$ et al also revealed that intubation status is an important independent predictor of mortality and should be used in statistical models that predict mortality for MVC patients ${ }^{(22)}$.

\section{Conclusions}

Extremities and pelvis injuries are the most common sites to be affected by blunt trauma. Only head and neck injuries had a significant association with mortality. A graded increase in the mortality was observed in association with the increase in both heart rate and shock index, and the decrease in systolic blood pressure and Glasgow coma scale. Comparable performances of the RTS, ISS, SI and MSI showed RTS as the poorest index, while SI and MSI were the best.

\section{References}

1. Kauvar DS, Lefering R, Wade CE. Impact of Hemorrhage on Trauma Outcome: An Overview of Epidemiology, Clinical Presentations and Therapeutic Considerations. J Trauma. 2006; 60(6): S3-11.

2. Butcher N, Balogh ZJ. The definition of polytrauma: the need for international consensus. Injury 2009;40 (s4): S12-22.

3. Søreide K. Epidemiology of major trauma. Br J Surg. 2009;96(7): 697-698.

4. Hoyt DB, Coimbra R. Trauma Systems. Surg Clin North Am. 2007;87(1): 21-35.

5. Kuhls DA, Malone DL, McCarter R, Napolitano LM. Predictors of mortality in adult trauma patients: the Physiologic trauma score is equivalent to the Trauma and Injury Severity Score. J Am Coll Surg 2002; 194(6): 695-704.

6. Baker SP. Injuries: The neglected epidemic: Stone lecture, 1985 American Trauma Society meeting. J Trauma 1987;27 (4): 343348. 
7. Guirguis EM, Hong C, Liu D, Watters JM, Baillie F, Mclntyre RW. Trauma outcome analysis of two Canadian centers using the TRISS method. J Trauma 1990;30 (4): 426429.

8. Murlidhar V, Roy N. Measuring trauma outcomes in India: An analysis based on TRISS methodology in a Mumbai university hospital. Injury. 2004;35 (4): 386- 390.

9. Totterman A, Madsen JE, Skaga NO, Røise O. Extra-peritoneal pelvic packing: a salvage procedure to control massive traumatic pelvic hemorrhage. J Trauma 2007;62 (4): 843-852.

10. Haider AH, Chang DC, Haut ER, Cornwell EE 3rd, Efron DT. Mechanism of Injury Predicts Patient Mortality and Impairment After Blunt Trauma. J Surg Res. 2009; 153 (1): $138-142$.

11. Curtis KA1, Mitchell RJ, Chong SS, Balogh ZJ, Reed DJ, Clark PT, D'Amours S, Black DA, Langcake ME, Taylor CB, McDougall P, Cameron PA. Injury trends and mortality in adult patients with major trauma in New South Wales. Med J Aust 2012; 197(4): 233237.

12. Copes W, Champion HR, Sacco WJ, Lawnick MM, Keast SL, Bain LW. The injury severity score revisited. J Trauma 1988;28 (1) : 69-77.

13. Sogut $O$, Sayhan $M B$, Gokdemira $M T$, Bolekenc ME, Ald B, Kosee R, Ozgonulf A, Kara HP. Analysis of Hospital Mortality and Epidemiology in Trauma Patients: A MultiCenter Study. J Curr Surg 2011;1(1): 19-24.

14. Singh J, Gupta G, Garg R, Gupta A. Evaluation of trauma and prediction of outcome using TRISS method. J Emerg Trauma Shock 2011; 4(4): 446-449.

15. Hsiao KY, Hsiao CT, Weng HH, Chen KH, Lin $L J$, Huang YM. Factors predicting mortality in victims of blunt trauma brain injury in emergency department settings. Emerg Med J 2008;2(10):670-673.

16. Ulvik A, Wentzel-Larsen $T$, Flaatten $H$. Trauma patients in the intensive care unit: short- and long-term survival and predictors of 30-day mortality. Acta Anaesthesiol Scand 2007;51(2): 171- 177.

17. Liu YC, Liu JH, Fang ZA, Shan GL, Xu J, Qi ZW, Zhu HD, Wang Z, Yu XZ. Modified shock index and mortality rate of emergency patients. World J Emerg Med 2012; 3(2): 114-117

18. Guyette F, Suffoletto B, Castillo JL, Quintero J, Callaway C, Puyana JC. Prehospital Serum Lactate as a Predictor of Outcomes in Trauma Patients: A Retrospective Observational Study. J Trauma 2011;70(4): ):782-786.

19. Hasler RM, Nuesch E, Jüni $P$, Bouamra $O$, Exadaktylos AK, Lecky F. Systolic blood pressure below $110 \mathrm{~mm} \mathrm{Hg}$ is associated with increased mortality in blunt major trauma patients: multicenter cohort study. Resuscitation 2011;82(9): 1202-1207.

20. Tinkoff GH, O'Connor RE. Validation of new trauma triage rules for trauma attending response to the emergency department. J Trauma 2002;52(6): 1153-1158.

21. Rabbani A, Moini M. Application of "Trauma and Injury Severity Score" and "A Severity Characterization of Trauma" Score to Trauma Patients in A Setting Different from "Major Trauma Outcome Study", Arch Iran Med. 2007;10(3): 383-386.

22. Hannan EL, Farrell LS, Bessey PQ, Cayten CG, Cooper A, Mottley L. Accounting for Intubation Status in Predicting Mortality for Victims of Motor Vehicle Crashes. J Trauma 2000;48(1): 76 - 81.

23. Bilgin NG, Mert E, Çamdeviren $M$. The usefulness of trauma scores in determining the life threatening condition of trauma victims for writing medical-legal reports. Emerg Med J 2005; 22 (11): 783-787.

24. King PM, Tucker WS, Waddell J, Brown T. Correlation of trauma scoring and outcome in a Canadian Trauma Centre. Can J Surg 1994;37(3): 185-188. 
